\title{
Jean-Antoine Colladon et la découverte de la loi de l'hybridation en 1821
}

\author{
Par G. De Morsier et M. Cramer
}

C'est à JeAn Rostand que revient le mérite d'avoir attiré l'attention sur les travaux de Colladon, de Genève. Dans son livre L'Atomisme en Biologie (1956), Jean Rostand consacre un chapitre à «Un précurseur de Mendel: le pharmacien Colladon?" Rostand a trouvé, dans un livre de W. F. EDWARDS (1776-1842), médecin et historien anglais vivant à Paris, intitulé : Des caractères physiologiques des races humaines considérés dans leur rapport avec l'histoire. Lettre à Amédée Thierry, auteur de l'Histoire des Gaulois et paru à Paris en 1829, le passage suivant: « M. Coladon (sic), pharmacien de Genève, pour multiplier les expériences sur les croisements de races et étendre nos idées sur ce sujet, élève un grand nombre de Souris blanches et de Souris grises. Il en étudia attentivement les mœurs et trouva le moyen de les faire produire en les croisant. Il commença alors une longue suite d'expériences en accouplant toujours une Souris grise à une Souris blanche. Quel résultat attendez-vous? Qu'il y ait eu souvent des mélanges. Non, jamais. Chaque individu des nouveaux produits était ou entièrement gris, ou entièrement blanc, avec les autres caractères de la race pure; point de métis, point de bigarrure, rien d'intermédiaire, enfin le type parfait de l'une ou de l'autre variété. Ce cas est extrême à la vérité; mais le précédent ne l'est pas moins; ainsi les deux procédés sont dans la nature; aucun ne règne exclusivement».

Rostand a trouvé également dans le Dictionnaire classique d'Histoire Naturelle à l'article Génération (1825), écrit par J.B. Dumas, les lignes suivantes: «Nous avons pu, grâce à la complaisante amitié de Colladon, membre distingué de la Société de Physique de Genève, soumettre à diverses reprises des liqueurs spermatiques de la Souris blanche et grise à un examen très soigné. L'identité de leurs animalcules est complète, soit pour la longueur absolue soit pour la forme du renflement céphalique qui, comme on l'a déjà dit, présente des caractères particuliers. » A cette époque, J.L.Prévost et J.B.Dumas faisaient leurs travaux célèbres sur les spermatozoïdes et sur la fécondation.

Toujours en 1956, H. GrüNEBERG, professeur de génétique à l'Université de Londres, dans sa leçon inaugurale du 12 novembre, se demande s'il est certain que Mendel n'a étudié que les petits pois jaunes et verts. Il rappelle 
que R.A. Fisher (1936) trouve que l'accord entre l'expérimentation et le calcul est trop étroit pour qu'il soit dû à la chance. Il pense que Mendel connaissait déjà la loi de l'hybridation avant d'expérimenter sur les petits pois et que cette dernière expérimentation lui en a donné seulement la meilleure démonstration. Il aurait ainsi omis de donner des résultats moins précis obtenus auparavant. D'autre part, Hugo Iltis, qui a fait une biographie de Mendel en 1924, dit que ce dernier élevait probablement des Souris grises et blanches avant 1856, année du début de ses travaux sur les petits pois. Malheureusement nous n'en aurons jamais la certitude, car les autorités ecclésiastiques ont fait brûler tous les papiers qu'a laissés le moine Gregor Mendel.

Pourquoi Mendel élevait-il des Souris ? Grüneberg pense que c'était pour reproduire et confirmer les expériences de Colladon. Le livre d'Edwards confirme aussi l'idée que les expériences de Colladon étaient connues à cette époque. La méthode de croisements répétés, employée en génétique, est donc une méthode « colladonienne » et non "mendélienne ».

En 1958, Jean Rostand, dans le Figaro Littéraire du 30 août, puis dans son volume Aux sources de la Biologie, revient sur la question dans un chapitre intitulé : "Colladon a-t-il influé sur Mendel? ». Depuis 1956, Jean Rostand a trouvé dans le célèbre mémoire de Jean-Louis Prévost et de J.B. Dumas sur la génération (1824), une allusion précieuse aux travaux de Colladon : «Nous avons pu, grâce à la complaisante amitié de M. Colladon, membre distingué de la Société de Physique de Genève, soumettre à diverses reprises les liqueurs spermatiques de la Souris blanche et de la Souris grise à un examen comparatif très soigné etc... » Le texte est identique à celui de Dumas cité plus haut. Mais les auteurs ajoutent: «A l'époque où nous étions occupés de cette recherche intéressante, M. Colladon fit connaître à la Société de Physique le résultat des observations qu'il faisait avec zèle et sagacité sur ces deux races de la Souris depuis quelques années. Les variétés blanche et grise s'accouplent sans difficulté; mais les petits qu'elles produisent n'offrent aucun mélange de nuance dans le pelage. Quelle que soit la combinaison de mâle et de femelle qu'on emploie, la génération qui en provient renferme des individus blancs et des individus gris en nombre variable; il ne se présente jamais de métis. Il en est de même si l'on forme de nouvelles associations avec les Souris blanches et les Souris grises de cette première génération; cette singularité se conserve encore à la troisième, et probablement elle persisterait malgré tous les mélanges successifs, puisque le nombre considérable de portées dont on a été témoin dans ces recherches 
n'a pu faire apercevoir d'altération dans la pureté des types gris et blancs originels. »

Colladon avait donc parfaitement vu les faits essentiels de l'hybridation raciale, la dominance d'un caractère indépendant du sexe des parents et la stabilité de ce caractère qui se montre pur à travers plusieurs générations, malgré les croisements répétés. Rostand a aussi montré que les expériences de Colladon étaient connues à cette époque d'après la note de Prévost et Dumas que nous venons de rapporter. Ses expériences sont citées aussi par Girou de Buzareingues en 1825 et par Antoine Dugès en 1839. Voici le texte de Prosper Lucas, auteur d'un gros Traité philosophique de l'Hérédité Naturelle dans l'état de santé et de Maladie du système nerveux, etc... en 2 volumes, publié en 1847, qui contient une quantité énorme de faits mais de valeur très inégale: "Chambon et Girou rapportent des espèces ovine et chevaline des cas identiques, Masch, de celle du cochon, Maupertuis, des chiens, Colladon, de Souris: les produits des Souris grises et des Souris blanches sont assez fréquemment ou tout blancs ou tout gris. Il en est de même des produits de mélange de la variété blanche et de la variété brune de l'espèce du cerf. »

Rostand a montré que Darwin connaissait aussi les expériences de Colladon sur les Souris et qu'il en parle dans son ouvrage De la variation des animaux et des Plantes à l'état domestique. Or, Iltis affirme que Mendel avait dans sa bibliothèque l'ouvrage de Darwin. Mendel ne pouvait donc ignorer les travaux de Colladon, mais il n'en a jamais parlé.

Nous avons pu consulter les comptes-rendus manuscrits des deux communications de Colladon, grâce à l'obligeance du Docteur JuNG, secrétaire de la Société de Physique et d'Histoire Naturelle de Genève. Ces comptesrendus n'ont jamais été publiés. Les voici :

« Séance générale du 3 mai 1821 - M. Deluc Président - M. Colladon lit une note.sur les Souris blanches, qu'il regarde comme une nouvelle espèce à cause de la constance de leur couleur.»

«Séance du 13 juin 1822 - Docteur Prévost Président - M. Colladon lit un mémoire sur les Souris blanches, qu'il considère comme une espèce particulière et sur lesquelles il a institué plusieurs expériences; il fonde son opinion sur l'absence de métis lorsqu'on accouple des Souris blanches avec des Souris grises; en ce cas, les petits sont de l'une ou de l'autre couleur; il insiste aussi sur la constance de la couleur blanche de génération en génération, qu'il a eu l'occasion d'observer depuis fort longtemps chez ces Souris, soit qu'il les conservât dans un endroit obscur ou au grand jour.» 
Il n'est pas sans intérêt de rapporter également le compte-rendu de la discussion qui a suivi cette communication du 13 juin 1822 :

«M. de Candolle ${ }^{1}$ ne pense pas que la Souris blanche soit une espèce mais bien une race. Cette question rentre dans celle des albinos.

»M. Colladon observe que les albinos chez les hommes ne se propagent point.

"M. Mayor ${ }^{2}$ rapporte des cas de propagation chez les hommes. A Chardonne, au-dessus de Vevey, on en rencontre deux générations dans une famille.

»M. de Candolle pense d'ailleurs que l'action produite chez l'homme par l'état d'albinos est trop puissante pour ne pas altérer la santé des individus.»

Ce compte-rendu très sommaire, rédigé sans doute par le Dr Gosse, secrétaire, confirme donc la note de Prévost, qui présidait la séance, et de Dumas, mais sans donner plus de renseignements. Nous ne savons pas si Colladon a constaté la répartition numérique des deux couleurs: $1 / 4$ de souris blanches pures, $1 / 4$ de souris grises pures, $1 / 2$ d'hybrides gris à la $2^{\mathrm{e}}$ génération. Il faut nous résigner à ne rien savoir de plus sur ces mémorables expériences, car le manuscrit du mémoire n'a pas été retrouvé (fig. 1).

Qui était ce Colladon?

Il y avait à Genève, à cette époque, trois Colladon naturalistes ou médecins. C'est par exclusion que nous pouvons arriver à identifier l'auteur des travaux sur l'hybridation.

Jean-Pierre Colladon (1769-1842) fait tout d'abord des études de lettres à Gœttingen, puis devient Docteur en médecine d'Edimburg. Il se fixe à Paris où il fut un des premiers à introduire la vaccine en France, puis revient à Genève où il fait partie de la Chambre de Santé. Il s'est occupé de la lutte contre le choléra et la fièvre jaune qui menaçaient Genève en 1821 et a publié une étude sur les Bains de St-Gervais. Il ne s'est occupé que de médecine pratique et a fait partie de la Société Médico-chirurgicale de Genève. Dans une communication retentissante faite à cette Société, il parle d'une dame de 63 ans, porteuse d'un goître volumineux, qui avait fait des accidents graves à la suite du traitement iodé que Coindet venait de découvrir. Ces renseignements nous sont donnés par Emile Thomas et Jean Olivier. Jean-Pierre Colladon n'a jamais été membre de la Société de Physique et d'Histoire Naturelle; il ne peut donc s'agir de lui.

1 Augustin-Pyramus de Candolle, 1778-1841, célèbre botaniste.

2 Dr Françors Mayor, 1779-1854. Il a découvert en 1818 l'auscultation des bruits du cœur du fœtus. 


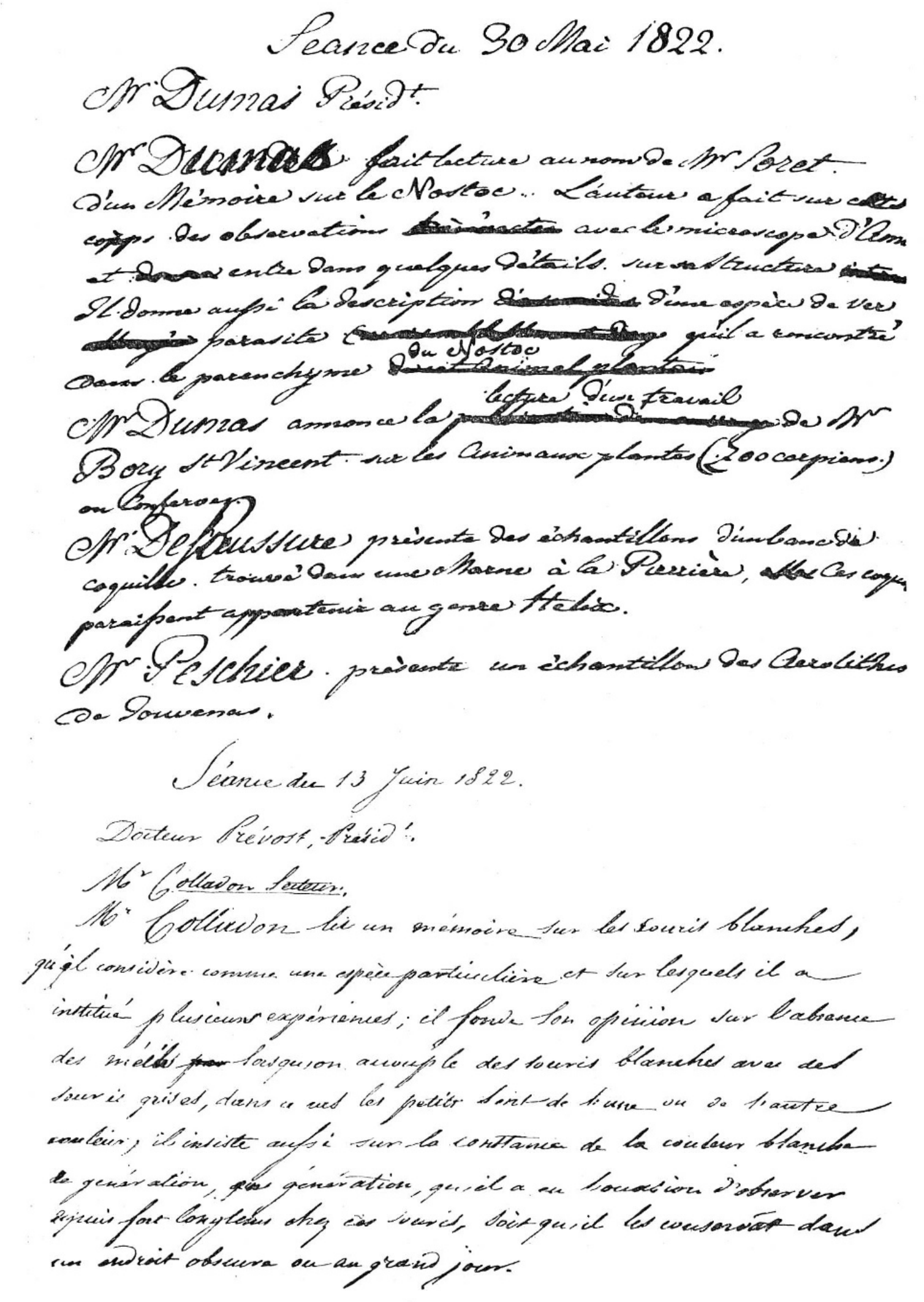

Fig. 1. Comptes-rendus des Séances de la Société de Physique et d'Histoire Naturelle de Genève, séances du 30 mai et du 13 juin 1822 
Louis-Théodore-Frédéric Colladon (1792-1862), fils du pharmacien et naturaliste Jean-Antoine, cousin de Jean-Pierre, Docteur en médecine de Montpellier en 1817, a publié en 1816 une Histoire naturelle et médicale des Casses et particulièrement de la Casse et des Sénés employés en médecine. Il a été proposé par Deluc comme « membre résident de la Société » le 12 juillet seulement et son nom figure dans la Liste des Membres à la fin de 1821. Il n'était donc pas membre de la Société de Physique lors de la première communication du 3 mai 1821, et il n'est pas possible que Prévost et Dumas aient parlé de lui comme un " membre distingué » de cette Société. D'autre part, dans les comptes-rendus de l'assemblée de la Société Helvétique des Sciences Naturelles qui s'est tenue à Bâle du 23 au 25 juillet 1821, on lit ceci :

«M. Colladon lit le résumé des travaux de la Société cantonale de Genève pendant l'année qui vient de se terminer. Il communique ensuite à la Société une lettre intéressante de son fils, M. le Dr Colladon (actuellement à Edimburg) contenant la description circonstanciée d'une descente dans une cloche de plongeur à la profondeur d'environ 20 pieds; les particularités de cette manière peu commune de voyager, ainsi que les observations physiologiques qui ont accompagné le récit excitaient beaucoup d'intérêt. »

Cette communication du Dr Frédéric Colladon, présentée par son père à la Société de Physique le 28 juin 1821, a été publiée la même année dans la Bibliothèque Universelle sous le titre : "Account of a descent in a diving Bell, etc. Relation d'une descente en mer dans la cloche de plongeur. " L'auteur raconte que les plongées ont été effectuées en septembre 1820 à Howth près de Dublin (Irlande). Frédéric Colladon était encore absent de Genève le 25 janvier 1821, car à cette date Jean-Antoine Colladon communique à la Société de Physique une lettre de son fils le Docteur Colladon où il est parlé de l'emploi médical des Cubèbes dans les Blennorrhées, dont les praticiens anglais ont reconnu l'efficacité. Il était très probablement en Angleterre encore en mai 1821 lors de la première communication sur l'hybridation, puisqu'il n'était pas rentré au mois de juillet 1821. Le $1^{\text {er }}$ novembre il n'était pas encore rentré car son père annonce à la Société de Physique l'envoi fait par Frédéric au Musée de «Minéraux des Isles Shetland et de plusieurs oiseaux d'Ecosse ». Le 4 octobre 1822, nouvelle lettre de Frédéric Colladon, lue par son père, sur les opérations géodésiques exécutées en Autriche. Frédéric a donc été absent de Genève de 1820 à 1823. Pour cetteraison aussi il n'est pas possible qu'il soit l'auteur des travaux sur l'hybridation

Un argument parle encore contre l'attribution de la communication à un Docteur en médecine. Dans les comptes-rendus et dans les mémoires de la 
Société de Physique de cette époque, les auteurs sont toujours affublés de leurs titres de Docteurs ou de Professeurs; or, lenom de Colladon est toujours précédé seulement de la lettre M., ce qui montre qu'il n'est pas Docteur en médecine (Fig. 2).

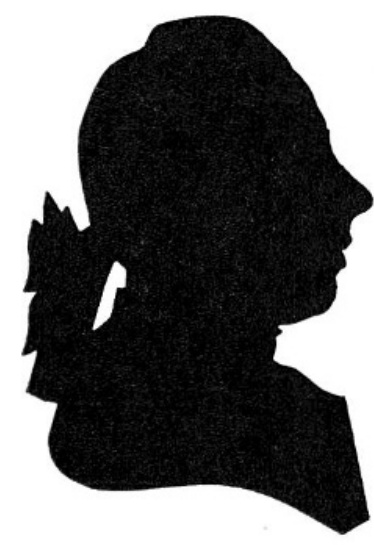

Fig. 2. Silhouette de Jean-Antorne Colladon à l'âge de 25 ans (Bibl. Publique et Universitaire de Genève)

Nous pouvons donc affirmer que l'auteur du fameux mémoire ne peut être que le pharmacien Jean-Antoine Colladon (1755-1830), père de Frédéric dont nous venons de parler. Nous avons des renseignements sur lui par un article nécrologique anonyme paru dans les Actes de la Société Helvétique des Sciences Naturelles, assemblée de St-Gall, 26-28 juillet 1830. En voici le texte :

«Monsieur Jean-Antoine Colladon était un des membres fondateurs de la Société Helvétique des Sciences Naturelles et faisait partie du Comité qui s'est réuni en 1814 chez M. Gosse pour organiser cette association et, dès lors, il s'y est rendu assez fréquemment et a souvent fait partie du Comité qui dirige les opérations.

" Il était né à Genève en 1756 et avait suivi la carrière de la pharmacie dans laquelle son père, Louis Colladon, avait déjà acquis quelque célébrité. Il aimait cet art soit pour les services qu'il rend à la science médicale, soit par ses relations avec la chimie et la botanique. Il l'exerçait avec soin et avec distinction et se plaisait à honorer la pharmacie de ses connaissances et la manière dont il l'exerçait. Il avait fait ses études en Allemagne et surtout à Berlin, et se tenait fort au courant des travaux des chimistes et pharmacologues allemands.

«M. Colladon a consacré une partie de son temps et de son influence pour favoriser dans Genève toutes les associations utiles : de concert avec Mrs de Saussure et Sénebier il a contribué à y fonder la Société des arts, institu- 
tion importante, qui a rendu une foule de services à l'industrie genevoise. De concert avec les mêmes savants et avec Mrs Pictet et Vaucher, il a contribué aussi à la formation de la Société de physique et d'histoire naturelle, qui est aujourd'hui la Société cantonale correspondante de la Soc. Helvétique. Dans ces diverses réunions M. Colladon se faisait remarquer par son zèle, son assiduité et le désir qu'il avait de répandre les connaissances utiles. Il y donnait souvent des traductions ou des extraits des mémoires scientifiques publiés en Allemagne. Il a dirigé, sous l'influence de la Société des arts, la fabrication de la gélatine des os, qui a rendu des services dans la disette de 1817.

« Il a présenté plusieurs mémoires à la Société de physique; nous citerons surtout : $1^{\circ}$ une analyse chimique de l'Hipophae rhamnoides, qui l'a occupé longtemps et dans les baies duquel il avait cru trouver un acide particulier; $2^{\circ}$ une analyse d'une terre de bruyère qu'on trouve au bois de Sauvablin près Lausanne, terre remarquable par la faculté qu'elle a de bleuir les fleurs de l'Hortensia; $3^{\circ}$ la part qu'il a eue à l'analyse de la matière singulière qui a coloré en rouge les eaux du lac de Morat et qui a été décrite dans les mémoires de la Société de Genève sous le nom d'Oscillatoria rubescens. M. Colladon a toujours aimé la botanique indigène et en particulier l'étude et la culture des plantes alpines. Il en a longtemps élevé chez lui, et à l'établissement du jardin de botanique il a pris beaucoup de soin pour y introduire ses plantes favorites. Il faisait partie de l'administration de cet établissement et suppléait le directeur lorsqu'il était absent. Il portait dans toutes les relations de la vie le calme qui résulte d'une bonté parfaite, d'un amour zélé pour le bien public et d'un désintéressement complet d'amour-propre. Il avait conservé toutes ses facultés jusque dans un âge avancé ; sa mort a été causée par un coup de froid qu'il a éprouvé pour avoir voulu, dans la rigueur de l'hiver dernier, assister à la cérémonie politique et religieuse du 31 décembre, dans laquelle Genève célèbre son retour à l'indépendance. Il est mort le 2 de janvier suivant. Ses qualités personnelles le faisaient chérir de tous ceux qui le connaissaient, et les regrets que sa mort a causés ont été universels. Il laisse un fils, M. Fréd. Colladon, qui exerce avec distinction la médecine à Paris et qui est connu dans la science par sa belle monographie du genre Cassia.»

L'article Colladon, Jean-Antoine, du Dictionnaire des Genevois et Vaudois, se borne à résumer ce texte.

En septembre 1915, le chimiste genevois Frédéric Reverdin a lu à la Société Suisse de Chimie des notes biographiques sur les chimistes ayant 
pris part à la fondation de la Société Helvétique des Sciences Naturelles. Voici ce qu'il écrit :

« Jean-Antoine Colladon a fait des études en Allemagne où il avait appris la chimie telle que Stahl et ses adhérents l'enseignaient... Aux côtés des H. A. Gosse et de Colladon, que leur activité comme pharmaciens nous a fait rapprocher l'un de l'autre, nous trouvons à la fondation de la Société Helvétique des Sciences Naturelles trois savants qui ont jeté sur la Genève du commencement du $\mathrm{XIX}^{\mathrm{e}}$ siècle un éclat tout particulier: Théodore de Saussure, Alexandre Marcet et Charles Gaspard de la Rive.»

Nous n'avons pas trouvé d'autres renseignements biographiques, ni de portrait de Colladon. La Bibliothèque Publique et Universitaire possède de lui une silhouette, alors qu'il était âgé de 25 ans (fig. 2). D'après Revilliod, la pharmacie Colladon se trouvait à la Grand-Rue $\mathrm{N}^{\circ}$ 7. Elle est devenue après la pharmacie Morin. La maison existe toujours, mais la façade a été remaniée ${ }^{3}$.

Colladon habitait à la campagne et cultivait dans son jardin un grand nombre de plantes. Il avait la passion de la science. Nous savons qu'il a aussi expérimenté sur des animaux. C'est lui qui a fourni à Prévost et Dumas les souris blanches dont ils avaient besoin pour leur travail Sur les animalcules spermatiques de divers animaux (1821). Dans les comptesrendus de la Société de Physique, à la date du 16 janvier 1823, nous lisons ceci : «M. Colladon cite quelques expériences faites avec de l'acide prussique sur des Souris et des chats. "Mais nous ne saurons jamais comment il est arrivé à cette idée géniale de croiser des Souris pendant plusieurs générations. Il ne pouvait pas comprendre lui-même l'importance considérable de sa découverte. Il ne faut pas oublier qu'en 1822 on ne savait rien sur le mécanisme de la fécondation. Une Science de l'hérédité, la Génétique, n'était pas concevable. On croyait avec Spallanzani (1785) que les spermatozoïdes étaient des sortes d'infusoires parasites qui ne jouaient aucun rôle dans la reproduction. Ce n'est que deux ans plus tard, en 1824, que J.L.Prévost et J. B. Dumas ont montré que le contact du spermatozoïde et de l'ovule est nécessaire. Et il a fallu attendre jusqu'en 1877 pour qu'un autre Genevois, Hermann Fol, montre la pénétration de la cellule mâle dans l'ovule. Après Colladon (1821), la Loi de l'Hybridation est tombée dans l'oubli. Après Mendel (1865), elle a été oubliée une deuxième fois ${ }^{4}$. Il a fallu at-

3 Cette maison a été démolie en été 1959.

4 Dans un travail qui vient de paraître, Elisabetr B. Gasking, de Melbourne, qui ne connaît pas les travaux de Colladon, cherche à expliquer pourquoi Mendel a été ignoré 
tendre les travaux des botanistes de Vries, Tchermak et Correns (1900) pour qu'on en comprenne enfin l'importance, c'est-à-dire 79 ans. C'est ainsi que lentement progresse la connaissance scientifique. Pour déployer ses effets, il ne suffit pas qu'une découverte soit faite, il faut encore qu'elle soit acceptée par la Science officielle de son époque et c'est souvent là que réside la plus grande difficulté 5 .

jusqu'en 1900. Elle étudie le rôle joué par Darwin dans cet oubli. (Why was Mendels Work ignored? Journal of the History of Ideas 20 [1959] 60-84.)

5 Ce travail était terminé lorsque le Dr René Tecoz, de Lausanne, a bien voulu nous envoyer, le 4 mai 1959, son mémoire intitulé «Un précurseur suisse de Mendel» paru dans le Bulletin de la Société Vaudoise des Sciences Naturelles du 28 février 1959, vol. 67, p. 127-32. Pour le Dr Tecoz, comme pour nous, c'est Jean-Antoine Colladon qui est l'auteur des travaux sur la reproduction des Souris. Nos conclusions concordantes montrent qu'il n'y a plus aucun doute possible sur ce point. Le Dr Tecoz montre qu'Edwards a assisté à la séance du 18 septembre 1823 de la Société de Physique, où il a pu voir Colladon.

\section{Sources}

Registre des Séances de la Société de Physique et d'Histoire Naturelle de Genève, du 6 janvier 1820 au 3 avril 1823 (manuscrit).

\section{Bibliographie}

Anonyme, Notice nécrologique sur Jean-Antoine Colladon, Actes de la Soc. Helv. Sciences Nat. (16 ${ }^{\mathrm{e}}$ assemblée, St-Gall, 26 à 28 juillet 1830). St-Gall, 1831, p. 108-9.

Colladon J. A., Résumé travaux de la Soc. Cant. de Genève. Notice des séances Soc. Helv. Sciences Nat. Bibl. Universelle 17 (1821) 828-9.

Colladon T., Account of a descent in a diving bell, etc. Bibl. Universelle 17 (1821) 828-9 (Genève).

Dumas J. B., Article «Génération » in Dictionnair e classique d'Histoire Naturelle, tome 7, p. 202, Paris 1825 .

EDWARDS W. F., Du caractère physiologique des races humaines considérées dans leur rapport avec l'histoire, etc., Paris 1829 (cité d'après Rostand).

Fisher R. A., Has Mendel's work been rediscovered? Ann. Science I (1936) 115-37 (cité d'après GRüNEBERG).

Gruneberg H., Genes in Mammalian Development. An Inaugural Lecture Delivered at University College, London (Lewis \& Co., London 1957, 17 p.).

Guyenot E., A propos d'un livre de Jean Rostand. Le Mystère de Colladon. Journal de Genève, 17-18 janvier 1959.

Itxis Hugo, Gregor Johann Mendel, Leben, Werk und Wirkung. Springer, Berlin 1924. 
Liste des membres de la Société de Physique et d'Histoire Naturelle de Genève, de 1790 à 1821. Mém. Soc. Phys. Hist. Nat. Genève, tome I, 1821, p. VIII-X.

Lucas Prosper, Traité philosophique et physiologique de l'Hérédité naturelle etc. Baillère, Paris 1847, tome I, p. 212.

Montet A. DE, Dictionnaire biographique des genevois et des vaudois etc. Art. Colladon, Jean-Antoine, Lausanne 1877.

Morsier (G. DE), Jean-Antoine Colladon et la découverte de la Loi de l'Hybridation en 1821 (Communication préliminaire). Soc. Méd. Genève 5 mai 1959 et Méd. et Hygiène, août 1959.

Ourvier J. Les origines de la Société Médicale de Genève et le Dr Jean François Coindet. $125^{\mathrm{e}}$ anniversaire de la Soc. Méd. de Genève, 24 juin 1948, p. 10.

Prévost J. L. et Dumas J. B., Essai sur les Animalcules spermatiques de divers animaux. Mémoires Soc. Phys. et Histoire Nat. de Genève. 1 (1821) 197.

Prévost J. L. et Dumas J. B., Observations relatives à l'appareil générateur des animaux mâles, etc. Ann. des Sciences Naturelles 1 (1824) 179-80 (Paris).

Reverdin F., Notice biographique sur J.A.Colladon. Actes de la Soc. Helv. Sc. Naturelles, Genève 12/15 septembre 1915. II lettre p. 283-4.

Revillod P., Physiciens et Naturalistes Genevois. Kündig, Genève 1942, p. 28 (photogr. d'une peinture de Grandpierre, 1879).

Rostand J., L'atomisme en biologie, Gallimard, Paris 1956, p. 202-8.

Rostand J., Aux sources de la Biologie, Gallimard, Paris 1958, p. 181-94.

Rostand J., Un précurseur de Johann Mendel. Le pharmacien suisse Colladon, «grandpère » de la génétique. Le Figaro littéraire, 30 août 1958.

Thомas E., La médecine (Genève au début du XIX $X^{e}$ siècle). Edition Ciba, 1937, p. 30 et 37. 